Check for updates

Cite this: RSC Adv., 2018, 8, 35534

Received 16th May 2018

Accepted 28th September 2018

DOI: $10.1039 / c 8 r a 04158 j$

rsc.li/rsc-advances

\section{The morphologies and fluorescence quantum yields of perylene diimide dye-doped PS and PHVB microspheres}

\author{
Jia Chen, $\dagger^{a}$ Shuoxun Jia, $\dagger^{a}$ Xiaohuan Ji, ${ }^{a}$ Mohamed Nourrein, ${ }^{a}$ Hengxue Xiang, ${ }^{a}$ \\ Zhe Zhou, ${ }^{a}$ Chien-Lung Wang, (D) *b Bin Sun (D) *a and Meifang Zhu*a
}

\begin{abstract}
Aggregation and continuous $\pi$-stacking have been the major obstacles hindering the fluorescence (FL) quantum yield $\left(\Phi_{\mathrm{F}}\right)$ of perylene diimide (PDI) derivatives in the condensed phase. To prepare polymer microspheres with nearly unity $\Phi_{\mathrm{F}}$, in this work a POSS functionalized PDI derivative, POSS-PDI-POSS (PPP), was applied as the red fluorophore of poly(3-hydroxylbutyrate-co-3-hydroxyvalerate) (PHBV) and polystyrene (PS) microspheres. The electrosprayed PPP/PHBV and PPP/PS microspheres have unique hollow structures. Moreover, they show bright red FL under a fluorescence microscope. A photophysical study of the microspheres indicates a significant role of the polymer matrix in disrupting the aggregation state and the $\Phi_{\mathrm{F}}$ of the embedded PPP fluorophore. Both the PPP/PHBV and the PPP/PS microspheres show higher $\Phi_{\mathrm{F}}$ than most PDI materials in the condensed phase. The PPP/PHBV microspheres show $\Phi_{\mathrm{F}}$ of $28 \%$, whereas the PPP/PS microspheres give nearly unity $\Phi_{\mathrm{F}}$.
\end{abstract}

\section{Introduction}

Polymeric microspheres are polymers that aggregate into spherical shapes with diameters at the nanometer $(\mathrm{nm})$ to micrometer $(\mu \mathrm{m})$ scale. Originally, macromolecular microspheres were obtained by emulsion polymerization, suspension polymerization and dispersion polymerization. With the progress of production techniques, scientists modified the electrospinning technique to develop facile electrospray methods for the preparation of polymer microspheres and nanospheres..$^{1-4}$ The electrospray technique facilitated the fabrication of multiphasic and multi-component polymer spheres and resulted in vigorous developments in the field of functional polymer microspheres. Polymer microspheres with hollow and coreshell structures are particularly useful for biological applications and nanotechnology. ${ }^{5}$ Although electrospray techniques provide ways to engineer the architecture of polymer particles, the properties of the particles still depend on the embedded active species.

Fluorescence (FL) is an important property for many nanomaterials. For example, FL detection is currently the dominant technique in the field of sensing technology. ${ }^{6-10}$ It facilitates the

\footnotetext{
${ }^{a}$ State Key Laboratory for Modification of Chemical Fibers and Polymer Materials, College of Materials Science and Engineering, Center for Advanced Low-Dimension Materials, Donghua University, 201620, Shanghai, P. R. China. E-mail: sunbin@ dhu.edu.cn; zmf@dhu.edu.cn

${ }^{b}$ Department of Applied Chemistry, National Chiao Tung University, 1001 Ta Hsueh Road, Hsin-Chu, 30010, Taiwan.E-mail: kclwang@nctu.edu.tw

$\dagger$ These authors contributed equally to the work.
}

detection of analytes present in biological samples by transducing the signals in optical imaging. ${ }^{11}$ Despite advances in the performance of conjugated molecules, applying conjugated molecules as fluorophores in polymer microspheres remains difficult, ${ }^{12,13}$ because the FL behaviors of conjugated molecules are strongly influenced by their aggregation states. ${ }^{14}$ Besides showing aggregation induced emission properties, ${ }^{15}$ the continuous $\pi$-stacking of conjugated molecules often causes decreases in FL quantum yield $\left(\Phi_{\mathrm{F}}\right)$. In studies of perylene diimide (PDI) based fluorophores, various molecular designs have been applied to reach high $\Phi_{\mathrm{F}}$ in different physical states. For example, Würthner assembled core-twisted PDI to prevent continuous $\pi$-stacking, which resulted in one-dimensional Jaggregates that delivered near unity $\Phi_{\mathrm{F}}$ in solution. ${ }^{16}$ In our group, by attaching two POSS molecular nanoparticles to the two N-positions of a PDI core, ${ }^{17}$ POSS-PDI-POSS (PPP in Scheme 1a) giant molecules are inclined to form unique dimer stacking (Scheme 1d) in the crystalline state, because the POSS units effectively inhibit the continuous $\pi$-packing of PDI. ${ }^{18}$ PPP thus shows $\Phi_{\mathrm{F}}$ of $100 \%$ in solution and $\Phi_{\mathrm{F}}$ of $48 \%$ in the crystalline phase.

Although nearly unity $\Phi_{\mathrm{F}}$ has been reported for PDI fluorophores in the solution state, when embedded into polymer microspheres, the highest $\Phi_{\mathrm{F}}$ of PDI-embedded polymer microspheres reported so far is $68 \%$, shown by PDI/PMMA (poly(methyl methacrylate)) microspheres. ${ }^{12,19}$ To enhance $\Phi_{\mathrm{F}}$ and to extend the applications of PDI microspheres, in this work, we blend PPP with polystyrene (PS) and poly(3-hydroxylbutyrate-co-3-hydroxyvalerate) (PHBV), respectively. Then, an electrospray technique is used to prepare the $\mathrm{PPP} /$ polymer 
(a)

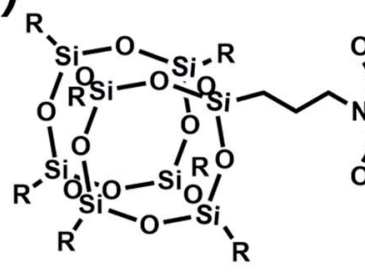

(c)

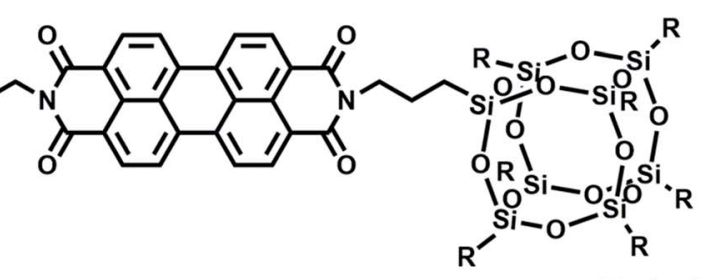

R=isobutyl (b)

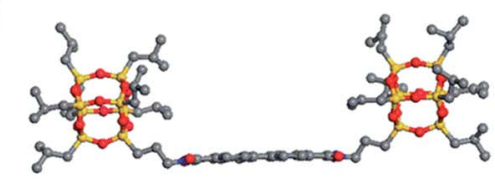

(d)

$M w=2.5 \times 10^{5}$<smiles>CC(C)Cc1ccccc1</smiles><smiles>[Y]C(C)(C)OC(CC)CC(=O)CC(C)OC(C)(C)C</smiles>

$M w=2.5 \times 10^{5}$

Scheme 1 Chemical structures of (a) POSS-PDI-POSS (PPP), (b) dimer packing diagram of PPP, (c) polystyrene (PS) and (d) poly(3-hydroxybutyrate-co-3-hydroxyvalerate) (PHBV).

microspheres. Significantly, PHBV is a biodegradable and biocompatible polyester synthesized by microorganisms. ${ }^{20-22}$ Via microscopic and photophysical experiments, a unique hollow sphere morphology and $\Phi_{\mathrm{F}}$ of nearly $100 \%$ were observed for the electrosprayed PPP/PS microspheres.

\section{Experimental}

\section{Synthesis}

POSS-PDI-POSS conjugates were synthesized according to literature procedures..$^{23,24}$ Unless otherwise noted, reagents and solvents were purchased as reagent grade and used without further purification. All reactions were carried out with standard glassware under an inert nitrogen atmosphere. Evaporation and concentration were carried out with water aspirator pressure and drying under vacuum at $10^{-2}$ Torr.

\section{Preparation of the spin-cast thin film}

The quartz plates were soaked in $10 \% \mathrm{NaOH}(\mathrm{aq})$ for $12 \mathrm{~h}$, sonicated with deionized water and ethanol for $10 \mathrm{~min}$, and then blow-dried with high purity nitrogen (99.9\%), before the film was cast by a $\mathrm{KW}-4 \mathrm{~A}$ spin coater. The $\mathrm{CHCl}_{3}$ solutions of PPP were dropped on the quartz plates and then fabricated at $500-2500 \mathrm{rpm}$ in $2-18$ seconds.

\section{Preparation of the electrostatic spraying solutions}

The $\mathrm{CHCl}_{3}$ solutions of the PPP/PS mixture were prepared by dissolving different ratios of PPP powders and PS slices in chloroform at $70{ }^{\circ} \mathrm{C}$. The solutions were stirred until all the solid compounds dissolved and the solution became clear. The concentration of the PPP/PS mixture in the solution was $2 \mathrm{wt} \%$, and the weight ratios of PPP/PS in chloroform were: 1/1000, $2 /$ $1000,4 / 1000$, and $8 / 1000(\mathrm{w} / \mathrm{w})$. For the $\mathrm{CHCl}_{3}$ solution of $\mathrm{PPP} / \mathrm{PHBV}$, the concentration of the PPP/PHBV mixture was also $2 \mathrm{wt} \%$ in the $\mathrm{CHCl}_{3}$ solution, and the weight ratios of PPP/PHBV in chloroform were: $1 / 1000,2 / 1000,4 / 1000$, and $8 / 1000(w / w)$.

\section{Preparation of fluorescence microspheres}

The solution was loaded into the injection syringe, as shown in Fig. 1, for the consequent electrostatic spraying process. The advancing speed of the syringe pump was $2 \mathrm{~mL} \mathrm{~h}^{-1}$, the receiving distance was $20 \mathrm{~cm}$, and the applied voltage was $15 \mathrm{kV}$ for the spraying process.

\section{General measurement}

Ultraviolet and visible (UV-vis) spectra of the solutions were recorded on a Lambda 35 (Perkin Elmer) spectrophotometer. UV-vis spectra of the films and microspheres were recorded on a Lambda 950 (Perkin Elmer) spectrophotometer. The excitation and emission spectra of the samples were recorded on a FP6600 steady-state fluorescence spectrophotometer (analysis range $200-800 \mathrm{~nm}$ with a resolution of $1 \mathrm{~nm}$ ). The solution sample was measured with a $2 \mathrm{~mm}$ or $10 \mathrm{~mm}$ cuvette, whereas the solid sample was detected with an integrating sphere.

Optical microscopy (OM) images were recorded by a YS2 optical microscope. The magnification of the sample was 400 times (eyepiece $10 \times$, objective $40 \times$ ). Fluorescence microscopy (FM) images were recorded by an Olympus BX-51 fluorescence microscope. The magnification of the sample was 400 times (eyepiece $10 \times$, objective $40 \times$ ) and the excitation wavelength was

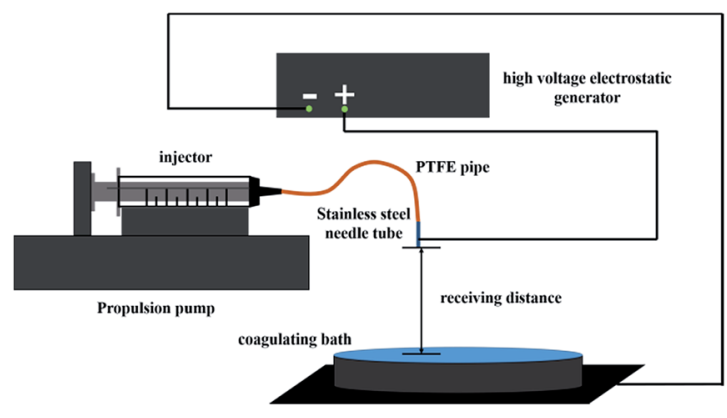

Fig. 1 Schematic illustration of the electrostatic spraying device. 
around 550-570 nm. Fluorescence quantum yield measurements were obtained on a JASCO FP-6600 spectrofluorometer equipped with a $60 \mathrm{~mm}$ diameter integrating sphere accessory and corresponding software. Microspheres were placed in a $10 \mathrm{~mm}$ diameter holder with a quartz window. The measured spectra were corrected for blanks by performing measurements for the reflectance standard $\left(\mathrm{Al}_{2} \mathrm{O}_{3}\right)$, with the sample emission spectrum measured under the same conditions. The excitation wavelength could be chosen from the 350-750 nm range of the spectrum. The morphologies of the microspheres were observed by a Japan HITACHI field emission scanning electron microscope (SEM). Transmission Electron Microscopy (TEM) observations were performed on a JEOL JEM-2010 transmission electron microscope with an accelerating voltage of $160 \mathrm{kV}$ and a Gatan-831 CCD camera.

\section{Results and discussion}

\section{Morphology of PPP/polymer microspheres}

The chemical structures of the materials used in the preparation of the FL microspheres are shown in Scheme 1. PPP was chosen as the fluorophore for the microspheres because it delivers high solid-state $\Phi_{\mathrm{F}}$ from its unique dimer packing. ${ }^{18}$ PS and PHBV were selected for the polymer matrices of the microspheres to verify the influence of the dispersion phase on the performance of the microspheres. The FL microspheres were fabricated via electrospraying $\mathrm{CHCl}_{3}$ solutions of $2 \mathrm{wt} \%$ PPP/PHBV or PPP/PS mixtures. A schematic illustration of the electrospray setup is shown in Fig. 1. The PPP/PHBV ratio affects the morphology of the microspheres. Above a PPP/PHBV ratio of $8 / 1000 \mathrm{w} / \mathrm{w}$, the size and shape of the microspheres cannot be well controlled. Nevertheless, as shown in Fig. 2, below a PPP/PHBV ratio of 4/1000 w/w, microspheres with welldefined shapes were prepared from the electrosprayed solutions.

Under the microscope, pristine PPP forms needle-like crystals in the drop-cast film, whereas the electrosprayed $\mathrm{CHCl}_{3}$ solution of PPP/PHBV results in microspheres, as shown in Fig. $2(\mathrm{c})$. Excited by green light $\left(\lambda_{\text {ex }}: 530 \mathrm{~nm}\right)$, both the needle crystals and the microspheres emit strong red light, as can be seen from the fluorescence microscopy (FM) micrographs in Fig. 2(b) and (c), suggesting that PPP is a great red fluorophore both in a pristine film and when it is dispersed in a polymer matrix. The SEM micrographs in Fig. 3 reveal the morphological details of microspheres prepared from the PPP/PHBV solutions.

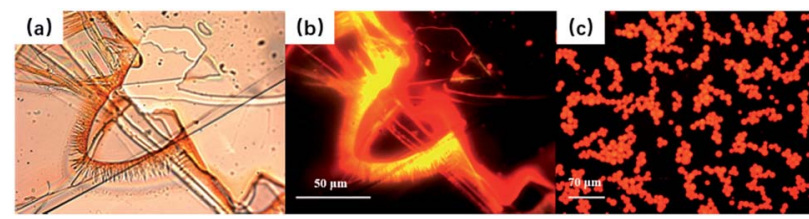

Fig. 2 (a) Optical micrograph and (b) fluorescence microscopy (FM) micrograph of a drop-cast thin film of PPP. (c) PPP/PHBV microspheres prepared via electrospray from a $\mathrm{CHCl}_{3}$ solution of PPP/PHBV $(1 / 1000 \mathrm{w} / \mathrm{w})$.

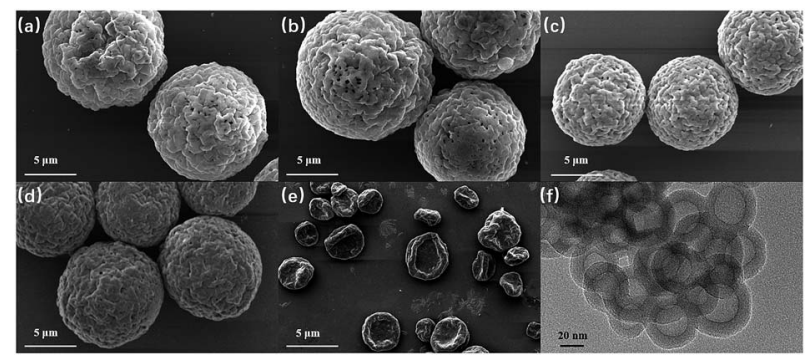

Fig. 3 SEM micrographs of the PPP/PHBV microspheres prepared from different PPP/PHBV ratios: (a) 4/1000 w/w, (b) 2/1000 w/w, (c) $1 /$ $1000 \mathrm{w} / \mathrm{w}$, and (d) 0.5/1000 w/w. (e) SEM micrograph of the PPP/PS (1/ $1000 \mathrm{w} / \mathrm{w}$ ) microspheres. (f) TEM micrograph of the PPP/PHBV (1/ $1000 \mathrm{w} / \mathrm{w})$ microspheres.

The diameters of the PPP/PHBV microspheres are around 8-10 $\mu \mathrm{m}$, and the microspheres have a rough surface. More interestingly, as shown in Fig. 3(f), the PPP/PHBV blend forms microspheres with hollow structures in the electrospray process. The unique morphology significantly increases the surface area of the microspheres and may enhance the performance of the microspheres in applications. Unlike the PPP/ PHBV microspheres, as shown in Fig. 3(e), the electrosprayed PPP/PS microparticles have a similar shape to erythrocytes and diameters of 10-25 $\mu \mathrm{m}$. These erythrocyte-like microparticles have a concave surface and many pores distributed on the surface. The morphology of the PPP/PS microparticles suggests that the PPP/PS blend also forms hollow particles during the electrospraying process. Nevertheless, the shell of the PPP/PS hollow particles was not strong enough to maintain the particle shape, so the PPP/PS macroparticles collapsed during the drying process. The formation mechanism of the rough and hollow microspheres of the PPP/PHBV and PPP/PS blends remains unclear. Why the $\mathrm{PPP} /$ polymer blends encapsulate solvents and form hollow particles is an interesting question to be answered and will be further investigated in a separate study.

\section{Photophysical properties of the PPP/PHBV microspheres}

The photophysical properties of the PPP/PHBV microspheres and the aggregation state of PPP in the microspheres were studied by UV-vis absorption and FL spectroscopy. In Fig. 4(a), compared to the spectrum of the PPP solution, the PPP thin film and $\mathrm{PPP} / \mathrm{PHBV}$ microspheres give UV-vis spectra with
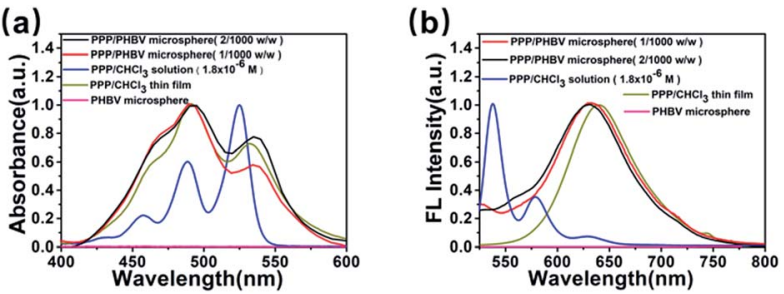

Fig. 4 (a) UV-vis absorption spectra and (b) FL spectra $\left(\lambda_{\text {ex }}=470 \mathrm{~nm}\right.$ ) of the PPP solution $\left(1.8 \times 10^{-6} \mathrm{M}, \mathrm{CHCl}_{3}\right)$, PPP thin film, and PPP/PHBV microspheres (1/1000 and 2/1000, w/w). 
broadened and red-shifted absorption bands. Previous studies indicate that in good solvents such as $\mathrm{CHCl}_{3}$, PPP behaves as individual molecules and delivers monomeric absorption characteristics that show sharp absorption bands at $460 \mathrm{~nm}$, $490 \mathrm{~nm}$ and $527 \mathrm{~nm}$, corresponding to the $0-2,0-1$, and $0-$ 0 electronic transitions of PDI. ${ }^{25,26}$ The broadened absorption bands suggest that PPP molecules are packed closely in the thin film and in the microspheres. In Fig. 4(b), monomeric PPP in solution shows a FL spectrum that is the mirror image of its absorption spectrum. On the contrary, the PPP film and the PPP/PHBV microspheres give broadened and red-shifted FL bands with $\lambda_{\mathrm{FL}}$ at $624 \mathrm{~nm}$ and $649 \mathrm{~nm}$, respectively. The PPP crystals emit FL at $624 \mathrm{~nm}$, but the fast-precipitated PPP powders show FL at 649 nm. ${ }^{18}$

Therefore, the FL band at $649 \mathrm{~nm}$ indicates that the PPP/ PHBV microspheres contain PPP molecules that have similar aggregation states to the rapidly-precipitated PPP powders. Although in both the crystal and powder states, PPP molecules form discontinuous dimeric packing rather than continuous $\pi-$ $\pi$ stacking because of the steric hindrance of the bulky POSS units, packing defects exist in the rapidly-formed PPP aggregates, and weaken the ability of the POSS units to confine the positions of the PDI units. The less well defined packing structures thus result in a higher possibility of non-fluorescence relaxation and a lower $\Phi_{\mathrm{F} .}{ }^{27}$ In Table 1 , the PPP/PBHV microspheres thus deliver $\Phi_{\mathrm{F}}$ values that are lower than the $\Phi_{\mathrm{F}}$ of the PPP crystal (48\%). Nevertheless, since the PHBV matrix dilutes the weight ratio of PPP, and the bulky POSS units prevent the PDI units from forming continuous $\pi-\pi$ stacking, the PPP dimers in the PPP/PHBV microspheres still deliver superior $\Phi_{\mathrm{F}}$ values $(23.9-28.5 \%)$ compared to the PPP precipitate $\left(\Phi_{\mathrm{F}}=\right.$ $17 \%$ ) and most of the PDI materials in the condensed phase.

\section{Photophysical properties of the PPP/PS microspheres}

In Fig. 5(a), the PPP/PS microspheres give absorption spectra that are very similar to those of the PPP/PHBV microspheres, suggesting that PPP molecules form aggregates in both the PHBV and PS matrices. However, the FL spectra of the PPP/PS microspheres are significantly different to those of the PPP/ PHBV microspheres. At the weight ratios of $1 / 1000(\mathrm{w} / \mathrm{w})$ and 2/1000 (w/w), the PPP/PS microspheres gave FL spectra similar to that of the PPP solution, as can be seen in Fig. 5(b). The results indicate that $\mathrm{PS}$ acts as a better matrix than PHBV to disperse PPP molecules and to disrupt $\pi$-stacking among the PDI units. Comparing the chemical structures of PHBV and PS, PS contains phenyl groups that have better miscibility with aromatic molecules. Thus, the more significant disturbance in the $\pi$-stacking in the PPP/PS microspheres can be attributed to

Table 1 The fluorescence quantum yields of the PPP/PHBV and PPP/ PS microspheres

\begin{tabular}{lccc}
\hline PPP/polymer (w/w) & $4 / 1000$ & $2 / 1000$ & $1 / 1000$ \\
\hline$\Phi_{\mathrm{F}}$ of PPP/PHBV & $28.3 \%$ & $28.5 \%$ & $23.9 \%$ \\
$\Phi_{\mathrm{F}}$ of PPP/PS & $57.8 \%$ & $78.4 \%$ & $99.7 \%$
\end{tabular}
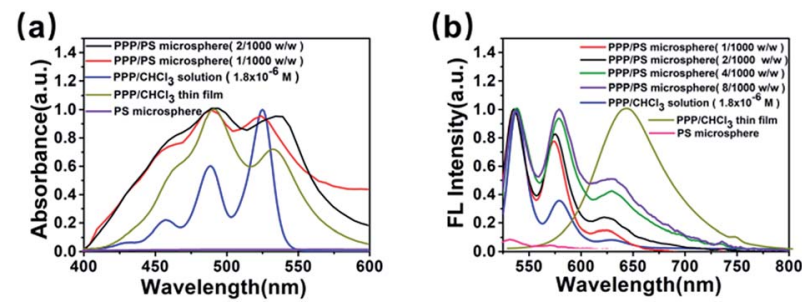

Fig. 5 (a) UV-vis absorption spectra and (b) FL spectra $\left(\lambda_{\text {ex }}=530 \mathrm{~nm}\right.$ ) of the PPP solution $\left(1.8 \times 10^{-6} \mathrm{M}, \mathrm{CHCl}_{3}\right)$, PPP thin film, and PPP/PS microspheres $(1 / 1000$ and $2 / 1000, w / w)$.

the better miscibility between the PPP fluorophore and the polymer matrix. Accompanying the disruption of $\pi$-stacking, the $1 / 1000 \mathrm{w} / \mathrm{w}$ PPP/PS microspheres show monomeric FL characteristics and deliver nearly $100 \% \Phi_{\mathrm{F}}$ (Table 1 ).

Table 1 also shows that $\Phi_{\mathrm{F}}$ of the PPP/PS microspheres decreases as the weight ratio of PPP increases. The red-shift of the FL band and the appearance of the FL shoulder at $\lambda_{\mathrm{FL}}=$ $649 \mathrm{~nm}$ suggest that PPP molecules form dimeric aggregation in the microspheres if the concentration of PPP is high enough. Nevertheless, the PPP/PS microspheres still deliver over $50 \% \Phi_{\mathrm{F}}$ at a weight ratio of $4 / 1000(\mathrm{w} / \mathrm{w})$. The results prove that combined with bulky POSS substituents and a suitable polymer matrix to disrupt the $\pi$-stacking, PDI fluorophores can deliver record high $\Phi_{\mathrm{F}}$ values when applied to polymer microspheres.

\section{Conclusions}

In this study, the morphologies and photophysical properties of PDI-embedded polymer microspheres were investigated. Hollow microspheres of PPP/PHBV and PPP/PS were successfully prepared using an electrospray technique. The microspheres show bright red FL under FM. UV-vis absorption and FL spectra of the microspheres indicate that the properties of the polymer matrix play a significant role in the aggregation state and $\Phi_{\mathrm{F}}$ of the embedded PDI fluorophore. Both the PPP/PHBV and the PPP/PS microspheres show higher $\Phi_{\mathrm{F}}$ values than most PDI materials in the condensed phase. The PPP/PHBV microspheres deliver $\Phi_{\mathrm{F}}$ of $28 \%$, whereas the PPP/PS microspheres give nearly unity $\Phi_{\mathrm{F}}$. The much higher $\Phi_{\mathrm{F}}$ of PPP/PS is attributed to the better ability of PS to disrupt the $\pi$-stacking of the PPP fluorophore. The results indicate that with the bulky POSS MNPs attached to the N-positions of the PDI unit, PPP is a red fluorophore that shows great potential for sensing and nanotechnology.

\section{Conflicts of interest}

There are no conflicts to declare.

\section{Acknowledgements}

This work was supported by the Science and Technology Commission of Shanghai Municipality (16JC1400700), the Program for Changjiang Scholars and Innovative Research 
Team in University (IRT-16R13) and the Ministry of Science and Technology, Taiwan, China (MOST 106-2221-E-009-130-MY3 Program).

\section{Notes and references}

1 N. Bock, M. A. Woodruff, D. W. Hutmacher and T. R. Dargaville, Polymers, 2011, 3, 131-149.

2 K. H. Roh, D. C. Martin and J. Lahann, Nat. Mater., 2005, 4, 759-763.

3 K. H. Roh, D. C. Martin and J. Lahann, J. Am. Chem. Soc., 2006, 128, 6796-6797.

4 H. Chen, Y. Zhao, Y. Song and L. Jiang, J. Am. Chem. Soc., 2008, 130, 7800.

5 G. L. Li, H. Mohwald and D. G. Shchukin, Chem. Soc. Rev., 2013, 42, 3628-3646.

6 K. Gardner, M. Aghajamali, S. Vagin, J. Pille, W. Morrish, J. G. C. Veinot, B. Rieger and A. Meldrum, Adv. Funct. Mater., 2018, 28, 1802759.

7 J. D. S. Hongying Zhu, I. M. White and X. Fan, Sensors, 2006, 2, 785.

8 J. R. Epstein, A. P. K. Leung, K.-H. Lee and D. R. Walt, Biosens. Bioelectron., 2003, 18, 541-546.

9 V. R. Sinha and A. Trehan, J. Controlled Release, 2003, 90, 261-280.

10 W. M. Lee, K. Grindle, T. Pappas, D. J. Marshall, M. J. Moser, E. L. Beaty, P. A. Shult, J. R. Prudent and J. E. Gern, J. Clin. Microbiol., 2007, 45, 2626-2634.

11 W. Zhong, Anal. Bioanal. Chem., 2009, 394, 47-59.

12 Y. Jiang and J. McNeill, Chem. Rev., 2017, 117, 838-859.

13 Y. Chen, H. Chen, H. Zhang and L. J. Fan, ACS Appl. Mater. Interfaces, 2015, 7, 26709-26715.
14 C. Li and H. Wonneberger, Adv. Mater., 2012, 24, 613-636.

15 Y. Hong, J. W. Y. Lam and B. Z. Tang, Chem. Soc. Rev., 2011, 40, 5361-5388.

16 T. E. Kaiser, H. Wang, V. Stepanenko and F. Würthner, Angew. Chem., Int. Ed., 2007, 46, 5541-5544.

17 W.-B. Zhang, X. Yu, C.-L. Wang, H.-J. Sun, I. F. Hsieh, Y. Li, X.-H. Dong, K. Yue, R. Van Horn and S. Z. D. Cheng, Macromolecules, 2014, 47, 1221-1239.

18 Y. Shao, G.-Z. Yin, X. Ren, X. Zhang, J. Wang, K. Guo, X. Li, C. Wesdemiotis, W.-B. Zhang, S. Yang, M. Zhu and B. Sun, RSC Adv., 2017, 7, 6530-6537.

19 H.-J. Ben, Y. Fan, Y.-J. Shi, R. Liu, Y. Chen, X.-K. Ren and S. Jiang, Dyes Pigm., 2017, 137, 584-592.

20 R. Vajtai, Springer Handbook of Nanomaterials, Springer Berlin Heidelberg, 2013, p. 263.

21 H. X. Xiang, S. H. Chen, Y. H. Cheng, Z. Zhou and M. F. Zhu, eXPRESS Polym. Lett., 2013, 7, 778-786.

22 P. J. Hocking, J. Macromol. Sci., Rev. Macromol. Chem. Phys., 1992, C32, 35-54.

23 H. Langhals, Heterocycles, 1995, 40, 477-500.

24 K. Balakrishnan, A. Datar, T. Naddo, J. L. Huang, R. Oitker, M. Yen, J. C. Zhao and L. Zang, J. Am. Chem. Soc., 2006, 128, 7390-7398.

25 R. Gvishi, R. Reisfeld and Z. Burshtein, Chem. Phys. Lett., 1993, 213, 338-344.

26 D. J. Liu, S. De Feyter, M. Cotlet, A. Stefan, U. M. Wiesler, A. Herrmann, D. Grebel-Koehler, J. Q. Qu, K. Mullen and F. C. De Schryver, Macromolecules, 2003, 36, 5918-5925.

27 F. Wuerthner, T. E. Kaiser and C. R. Saha-Moeller, Angew. Chem., Int. Ed., 2011, 50, 3376-3410. 